\title{
Las arañas del bosque mesófilo de montaña de la Reserva de la Biosfera Volcán Tacaná, Chiapas, México
}

\section{Spiders of the cloud montane forest of the Biosphere Reserve Volcán Tacaná, Chiapas, Mexico}

\author{
Guillermo Ibarra-Núñez ${ }^{1}$, Julieta Maya-Morales ${ }^{1}$ y David Chamé-Vázquez ${ }^{2}$ \\ ${ }^{l}$ El Colegio de la Frontera Sur, Unidad Tapachula. Carr. Antiguo Aeropuerto Km 2.5, 30700 Tapachula, Chiapas, México. \\ ${ }^{2}$ Universidad de Ciencias y Artes de Chiapas, Escuela de Biología. Libramiento Norte Poniente s/n, 29039 Tuxtla Gutiérrez, Chiapas, México. \\ 囚gibarra@ecosur.mx
}

\begin{abstract}
Resumen. Se realizó un estudio para conocer la fauna de arañas del suelo y del sotobosque en 2 sitios de bosque mesófilo de montaña de la Reserva de la Biosfera Volcán Tacaná. Las arañas del suelo se recolectaron con trampas de caída y mediante el procesamiento de hojarasca en embudos de Berlese; para las arañas del sotobosque se realizaron recolectas directas, por golpeo (red y manta) y con trampas de refugio. En total se registraron 32 familias, 99 géneros y 151 especies, que representan $32.5 \%$ de la araneofauna del estado de Chiapas. Se encontraron 7 registros nuevos de géneros y 12 de especies para México, así como de 6 géneros y 9 especies para el estado de Chiapas. Las familias con mayor riqueza fueron Theridiidae, Linyphiidae, Anyphaenidae, Araneidae y Salticidae. En el suelo se registraron 75 especies, 57 géneros y 26 familias mientras que en el sotobosque se encontraron 112 especies, 74 géneros y 22 familias; 36 especies fueron comunes a los 2 estratos, en tanto que 39 fueron exclusivas del suelo y 76 del sotobosque. La complementariedad entre los 2 estratos fue de $76.2 \%$.
\end{abstract}

Palabras clave: Araneae, riqueza de especies, suelo, sotobosque.

\begin{abstract}
A spider inventory from forest floor and understory in 2 sites of the cloud montane forest of the Biosphere Reserve Volcán Tacaná, Chiapas was undertaken. Soil spiders were collected using pitfall traps and processing litter in Berlese funnels; understory spiders were captured by hand collecting, sweeping, beating, and with shelter traps. Collected specimens correspond to 32 families, 99 genera and 151 species, which represent $32.5 \%$ of the spiders recorded for the state of Chiapas. There are 7 new records of genera and 12 of species for Mexico and 6 genera and 9 species for Chiapas. The families with most species richness were Theridiidae, Linyphiidae, Anyphaenidae, Araneidae and Salticidae. In the forest floor were recorded 75 species, 57 genera and 26 families and in the understory 112 species, 74 genera and 22 families. Both strata shared 36 species, and 39 and 76 species were exclusive to the forest floor and to the understory respectively. Complementarity between the 2 strata was $76.2 \%$.
\end{abstract}

Key words: Araneae, species richness, forest floor, understory.

\section{Introducción}

La región Mesoamericana está considerada una de las áreas con alta biodiversidad y endemismos bajo severa amenaza de destrucción del hábitat (hotspots; Myers et al., 2000). En dicha región, el bosque mesófilo de montaña (BMM) representa el tipo de vegetación con mayor riqueza de especies por superficie de área, y el más amenazado junto con el bosque tropical perennifolio (Acosta, 2004). En México, el BMM ocupa menos del 1\% de la superficie, pero es de gran importancia para los ciclos hidrológicos de las cuencas a las que se encuentra asociado, y al mismo tiempo es uno de los hábitats más frágiles (Toledo, 2010).

Raramente se han llevado a cabo inventarios de especies y análisis de endemismos de invertebrados en el BMM

Recibido: 15 enero 2011; aceptado: 08 marzo 2011
(Anderson y Ashe, 2000; Bubb et al., 2004). Entre los diferentes invertebrados terrestres, las arañas se caracterizan por presentar alta diversidad taxonómica y por exhibir respuestas a cambios ambientales específicos, sea el taxón o el gremio (Rubio et al., 2008), que incluyen diferencias en composición y riqueza entre los distintos estratos de un hábitat (Sørensen, 2003) debido a sus hábitos de caza y a la influencia de la complejidad estructural de la vegetación.

En la actualidad se conocen 41719 especies de arañas pertenecientes a 3802 géneros y 109 familias (Platnick, 2010). La cuenta para México alcanzó 2506 especies (Jiménez, 1996) y 464 para el estado de Chiapas, pertenecientes a 281 géneros y 58 familias (García-Villafuerte, 2009). En México, la fauna de arañas se ha estudiado en distintos tipos de hábitats naturales, como matorral xerófilo (Ibarra-Núñez, 1979), bosque de pino-encino (Medina, 2002), humedales (Llinas y Jiménez, 2004; Medina, 2005; 
Santos, 2005), selva alta perennifolia (Valdez et al., 2005) y selva baja caducifolia (Álvarez, 1999; Castelo, 2000; Durán, 2000; Nieto, 2000; Correa, 2001; Corcuera y Jiménez, 2009); sin embargo, los estudios encaminados a conocer la diversidad de arañas en los BMM del país son escasos (Santos, 2001; Ibarra-Núñez y Chamé-Vázquez, 2009; Maya-Morales, 2010). Por lo anterior, el objetivo de este trabajo fue dar a conocer la araneofauna del suelo y del sotobosque del BMM de la Reserva de la Biosfera Volcán Tacaná.

\section{Materiales y métodos}

Área de estudio. Se seleccionaron 2 sitios pertenecientes al ejido Talquián en el municipio de Unión Juárez, Chiapas dentro de la Reserva de la Biosfera Volcán Tacaná, próximos a la localidad de Chiquihuites (Fig. 1) y distantes entre sí sólo unos 800 metros. El primer sitio está ubicado en las coordenadas $15^{\circ} 05^{\prime} 14.8^{\prime \prime} \mathrm{N}$ y $92^{\circ} 05^{\prime} 55^{\prime} \mathrm{O}$, a $2021 \mathrm{~m}$ y el segundo, en las coordenadas $15^{\circ} 05^{\prime} 37.8^{\prime \prime} \mathrm{N}$ y $92^{\circ} 06^{\prime} 05.7$ 'O, a $2048 \mathrm{~m}$. En esta área se presenta un clima de tipo $\mathrm{C}(\mathrm{m})(\mathrm{w}$ ") ig, templado húmedo con régimen de lluvias de verano concentradas de mayo a octubre, con precipitaciones anuales promedio cercanas a $4000 \mathrm{~mm}$, temperatura media anual menor a $18^{\circ} \mathrm{C}$, que varía menos de $5^{\circ} \mathrm{C}$ a lo largo del año y con el mes más caliente antes de junio (Cardoso, 1979; SPP, 1981).

Trabajo de campo. Se llevó a cabo en 2 etapas, cada una correspondiente a un estrato. La primera parte de la recolección de las arañas del suelo se realizó de diciembre de 2006 a mayo del 2007, con 2 muestreos por mes; la segunda, fue de junio a noviembre del 2007 , con 1 muestreo por mes. En cada sitio se trazaron 2 transectos paralelos de $50 \mathrm{~m}$ de largo, separados entre sí por $25 \mathrm{~m}$ o más y en

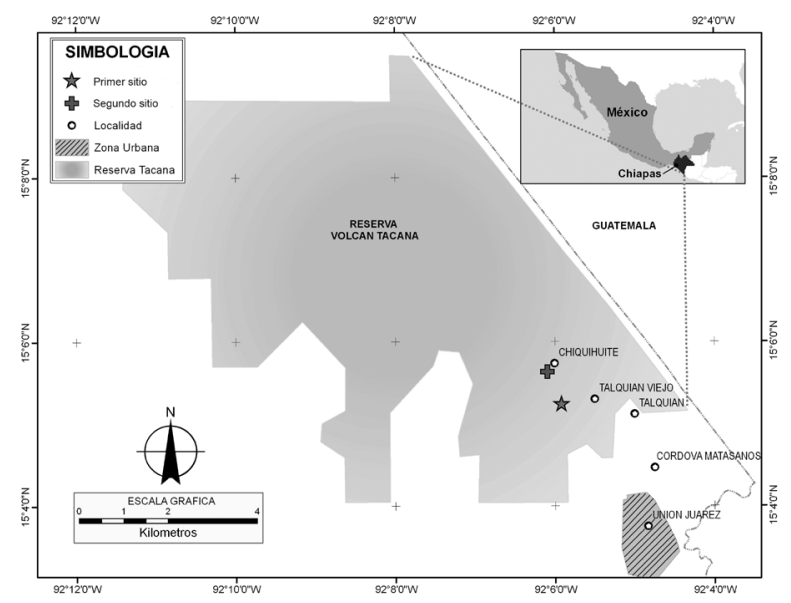

Figura 1. Localización de los sitios de estudio dentro de la Reserva de la Biosfera Volcán Tacaná, Chiapas, México. cada uno se aplicaron 2 técnicas de muestreo: trampas de caída y recolección de hojarasca. Para la primera, en cada muestreo se colocaron 10 trampas por transecto, separadas $5 \mathrm{~m}$ entre sí; estas trampas consistieron en un recipiente de plástico de $250 \mathrm{ml}$ de capacidad sujeto dentro de un tubo de PVC de $10 \mathrm{~cm}$ de largo y un diámetro de $7.5 \mathrm{~cm}$; cada una contenía $150 \mathrm{ml}$ de una solución acuosa de etilenglicol al $50 \%$ como fijador y preservador; se enterraron con el borde superior a nivel del suelo sin ninguna cubierta de protección (Mallis y Hurd, 2005; Schmidt et al., 2006); a las 72 horas de su colocación se extrajeron y el contenido se vació en frascos pet, etiquetados con sus correspondientes datos de recolecta. Para la segunda técnica, en cada muestreo se seleccionaron al azar 5 puntos por transecto (alejados de las trampas de caída y evitando la repetición de áreas previamente muestreadas), para recoger la hojarasca acumulada en una área de $50 \times 50 \mathrm{~cm}$ y colocarla en bolsas de plástico a las cuales se añadió una etiqueta con los datos de recolecta; estas bolsas se transportaron el mismo día al laboratorio, donde la hojarasca se procesó en embudos de Berlese por 2 a 4 días, según el contenido de humedad.

La recoleccción de arañas del sotobosque se llevó a cabo mediante 12 muestreos durante la época de secas (enero a marzo) y lluvias (junio a agosto) en 2009. En cada sitio se trazaron 3 transectos de $50 \mathrm{~m}$ de largo, separados por $25 \mathrm{~m}$ o más, en los cuales se establecieron 5 puntos de muestreo por transecto, cada punto de forma circular de 5 $\mathrm{m}$ de diámetro (19.6 $\mathrm{m}^{2}$ de superficie). Las arañas fueron capturadas mediante 3 métodos: recolecta directa, golpeo (red y manta) y trampas de refugio. En cada transecto se aplicó un método diferente, de manera que los 3 métodos fueron empleados en cada sitio y muestreo. La recolecta directa consistió en localizar visualmente y capturar los ejemplares en cada punto de muestreo. Se invirtieron 20 minutos de revisión de la vegetación por punto de muestreo, 10 minutos para revisión de la vegetación alta (de la cintura hacia arriba hasta el alcance máximo del brazo) y 10 minutos para revisión de la vegetación baja (de la cintura hacia abajo) (modificado de Coddington et al., 1991). El golpeo consistió en sacudir o golpear la vegetación con red o bastón. El método de red se aplicó a la vegetación baja (30 golpes) y la manta de golpeo consistió en golpear o sacudir con un bastón la vegetación alta colocando abajo una manta blanca de $1 \mathrm{~m}^{2}$ para recuperar el material caído (30 golpes). Las trampas de refugio (cuyo objetivo es capturar arañas que no están activas de día) se elaboraron con hojas caídas de la vegetación local, enrolladas e introducidas dentro de tubos de plástico pequeños $(2 \mathrm{~cm}$ de largo) de 2 tamaños de diámetro (1 y $1.5 \mathrm{~cm})$ que llevaban por fuera un clip parcialmente desdoblado, a manera de gancho, para sujetarlas de la vegetación de cada punto de 
muestreo. Se colocaron 200 trampas (100 por sitio), con 2 semanas de anticipación a cada muestreo, 20 trampas (10 de cada tamaño) por punto de muestreo en los troncos de los árboles y las ramas de los arbustos y las herbáceas, a una altura no mayor a $2 \mathrm{~m}$.

Los ejemplares recolectados de ambos estratos fueron conservados en etanol al 80\% y depositados en la Colección de Arácnidos del Sureste de México (ECOTAAR) de El Colegio de la Frontera Sur, Unidad Tapachula y se identificaron con ayuda de literatura especializada. Se empleó el índice de Colwell y Coddington (1994) para conocer la complementariedad de la composición entre los ensambles de suelo y sotobosque. Para la recolecta de ejemplares se contó con 2 permisos de la SEMARNAT (SGPA/ DGVS/03564/06 y SGPA/DGVS/00034/09).

\section{Resultados}

Se registraron 151 especies de arañas pertenecientes a 99 géneros y 32 familias (Apéndice 1). La lista incluye 54 especies (35.8\%) plenamente identificadas, 49 (32.4\%) que se han confirmado como especies nuevas (aún no descritas), 19 (12.6\%) que fueron determinadas como afines a especies descritas ya que requieren mayor análisis para confirmar estas determinaciones, 15 (9.9\%) especies que no pudieron determinarse más que a familia o género, por tratarse de ejemplares juveniles y 14 (9.3\%) no pudieron ser ubicadas por ser parte de grupos poco trabajados o porque es posible que sean especies o géneros nuevos. Las familias con mayor riqueza de especies fueron Theridiidae con 29, Linyphiidae con 20, Anyphaenidae y Araneidae con 14 cada una y Salticidae con 13 (Fig. 2).

En el suelo se registraron 75 especies, 57 géneros y 26 familias mientras que en el sotobosque se encontraron 112 especies, 74 géneros y 22 familias. Los 2 estratos compartieron 36 especies, 39 fueron exclusivas de suelo y 76 de sotobosque (Fig. 3). De estos datos se deriva que la complementariedad entre los estratos es de $76.2 \%$. De la fauna encontrada, 7 géneros y 12 especies son registros nuevos para México (Figs. 4 y 5). Adicionalmente se obtuvo un género nuevo para la ciencia de la familia Linyphiidae (en descripción). Para el estado de Chiapas se presentan nuevos registros de 6 géneros y 9 especies (Fig. 6).

\section{Discusión}

Los resultados de este trabajo contribuyen a incrementar el número de taxones registrados para México y para el estado de Chiapas. En el primer caso, Fissiscapus Millidge, 1991 se registró previamente en Colombia y Ecuador; Paraletes Millidge, 1991 en Brasil y Perú; Pescennina Simon, 1903 en Venezuela, y Wirada Keyserling, 1886 en

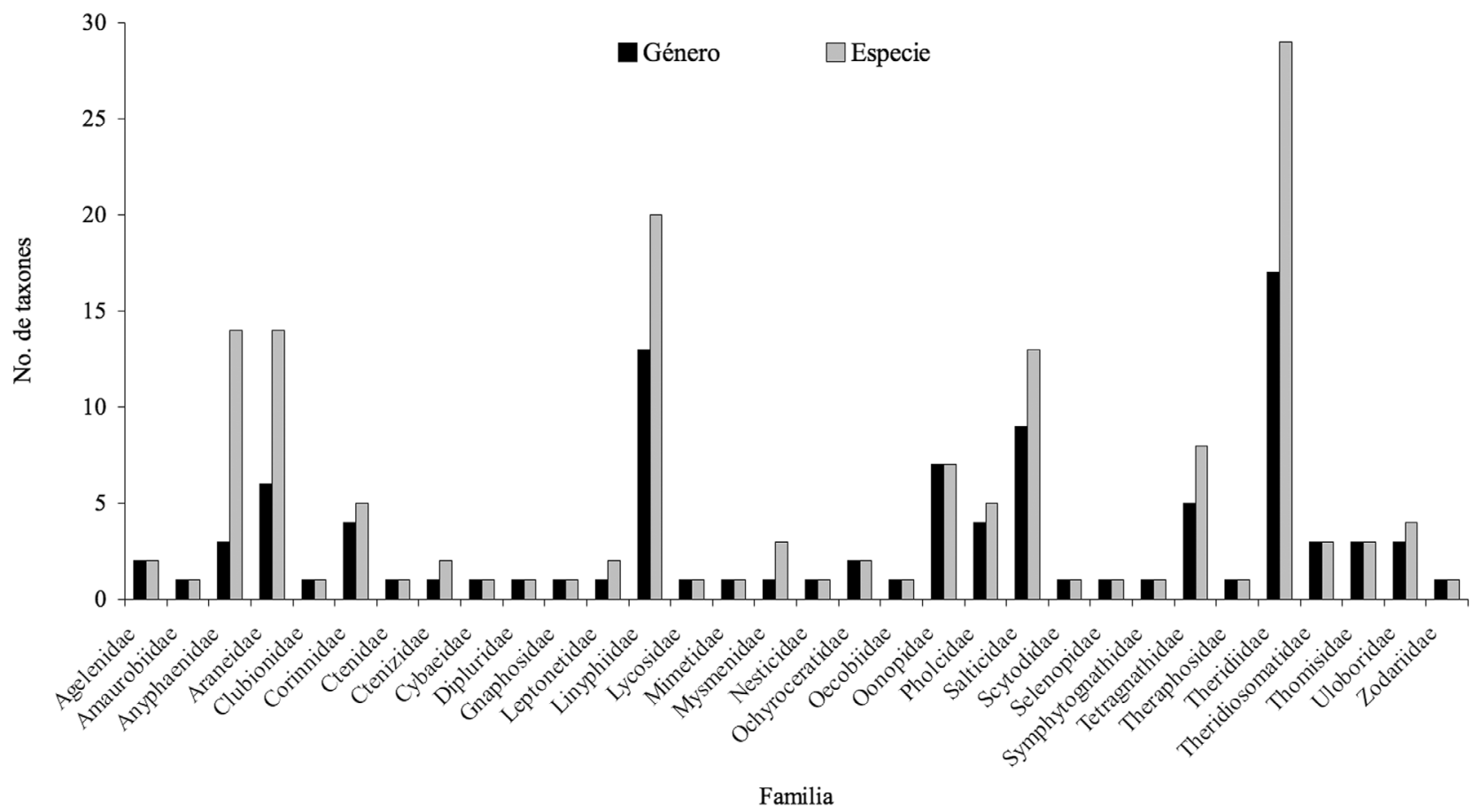

Figura 2. Riqueza de géneros y especies de cada familia de arañas recolectadas en el bosque mesófilo de la Reserva de la Biosfera Volcán Tacaná, Chiapas, México. 


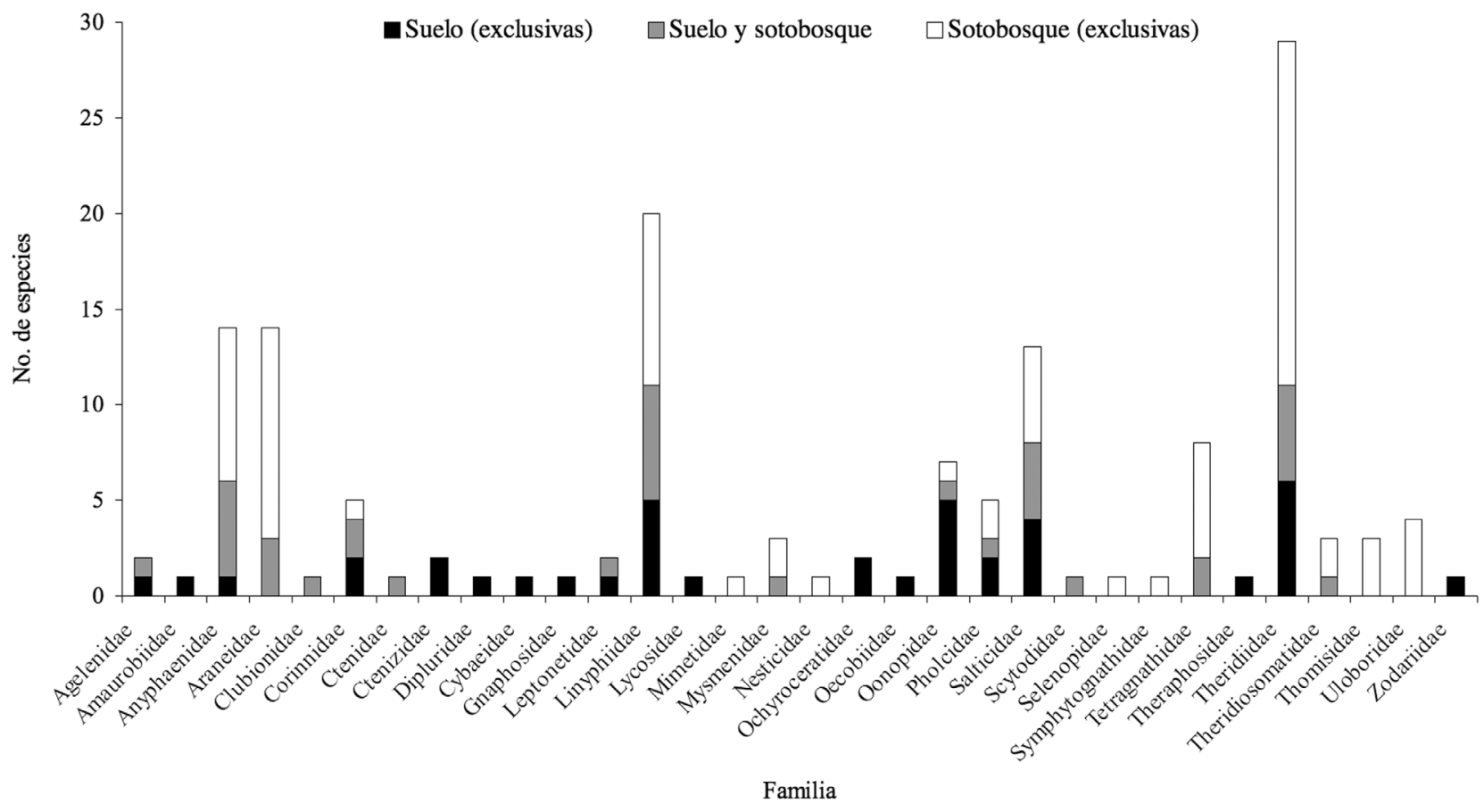

Figura 3. Riqueza de especies de arañas por estrato y familia en el bosque mesófilo de la Reserva de la Biosfera Volcán Tacaná, Chiapas, México.

Venezuela, Brasil, Ecuador y Perú; para Sidymella Strand, 1942, las especies americanas se registran en Colombia, Brasil, Uruguay y Argentina. Josa nigrifrons (Simon, 1897), registrada de Costa Rica a Bolivia y Tennesseellum formica (Emerton, 1882) de los Estados Unidos a Alaska y en las Islas Marshall, se consideran nuevos registros tanto en nivel genérico como específico. Como registros nuevos en el nivel de especie están Rualena simplex (F. O. P.-Cambridge, 1902) y Ummidia zebrina (F. O. P.Cambridge, 1897), colectadas previamente en Guatemala, U. zilchi Kraus, 1955 en El Salvador, Grammonota teresta Chickering, 1970 en Panamá y Colombia, Leucauge acuminata (O. P.-Cambridge, 1889) de Guatemala a Panamá, Phycosoma altum (Keyserling, 1886) de Panamá a Brasil y en Hawai, Thymoites puer (Mello-Leitão, 1941) en Brasil y Argentina, Wamba congener O. P.-Cambridge, 1896 en Estados Unidos y de las Antillas a Brasil, Tmarus vitusus Chickering, 1965 en Panamá y Philoponella tingens (Chamberlin e Ivie, 1936) de Costa Rica a Colombia (Platnick, 2010) (Figs. 4 y 5).

Los nuevos registros de géneros para el estado de Chiapas son Piabuna Chamberlin e Ivie, 1933 con distribución previamente registrada en Coahuila (Gertsch y Davis, 1940), Naphrys Edwards, 2003 en Coahuila, Nuevo León y Tamaulipas (Richman, 1981) y Paramarpissa F. O. P.Cambridge, 1901 en Sonora, Chihuahua, Nayarit, Jalisco,
Michoacán, Distrito Federal, Puebla y Tabasco (Logunov y Cutler, 1999). Melpomene transversa (F. O. P.- Cambridge, 1902) registrada en Guerrero, Selenyphantes longispinosus (O. P.-Cambridge, 1896) en Veracruz y Scaphiella cocona Platnick y Dupérré, 2010 en Tabasco son nuevos registros de género y especie para el estado. Mangora ixtapan Levi, 2005 se registró previamente en Michoacán, Linyphia nigrita (F. O. P.-Cambridge, 1902) en Hidalgo, Mermessus trilobatus (Emerton, 1882) en Veracruz, Dysderina plena O. P.-Cambridge 1894 y Leucauge simplex F. O. P.-Cambridge, 1903 en Tabasco y Misumenoides annulipes (O. P.-Cambridge, 1891) en Jalisco (Fig. 6).

Otro aspecto destacado de este estudio es la elevada proporción de especies encontradas no descritas (32.4\% del total de especies), que se debe en parte a la falta de estudios taxonómicos de arañas en los BMM, y en parte al aislamiento de este tipo de hábitats, que origina una considerable proporción de endemismos.

Por otro lado, la riqueza en el sitio estudiado es alta y comparable con la registrada en otros hábitats tropicales. Un estudio similar llevado a cabo en Tanzania en BMM (Sørensen, 2003), que incluyó además el dosel de los árboles, registró 175 especies de arañas en 33 familias. En cada estrato estudiado, la riqueza de especies observada en el suelo fue algo menor que la registrada en Tanzania (75 vs. 96), pero la del sotobosque fue muy similar (112 vs.114), 

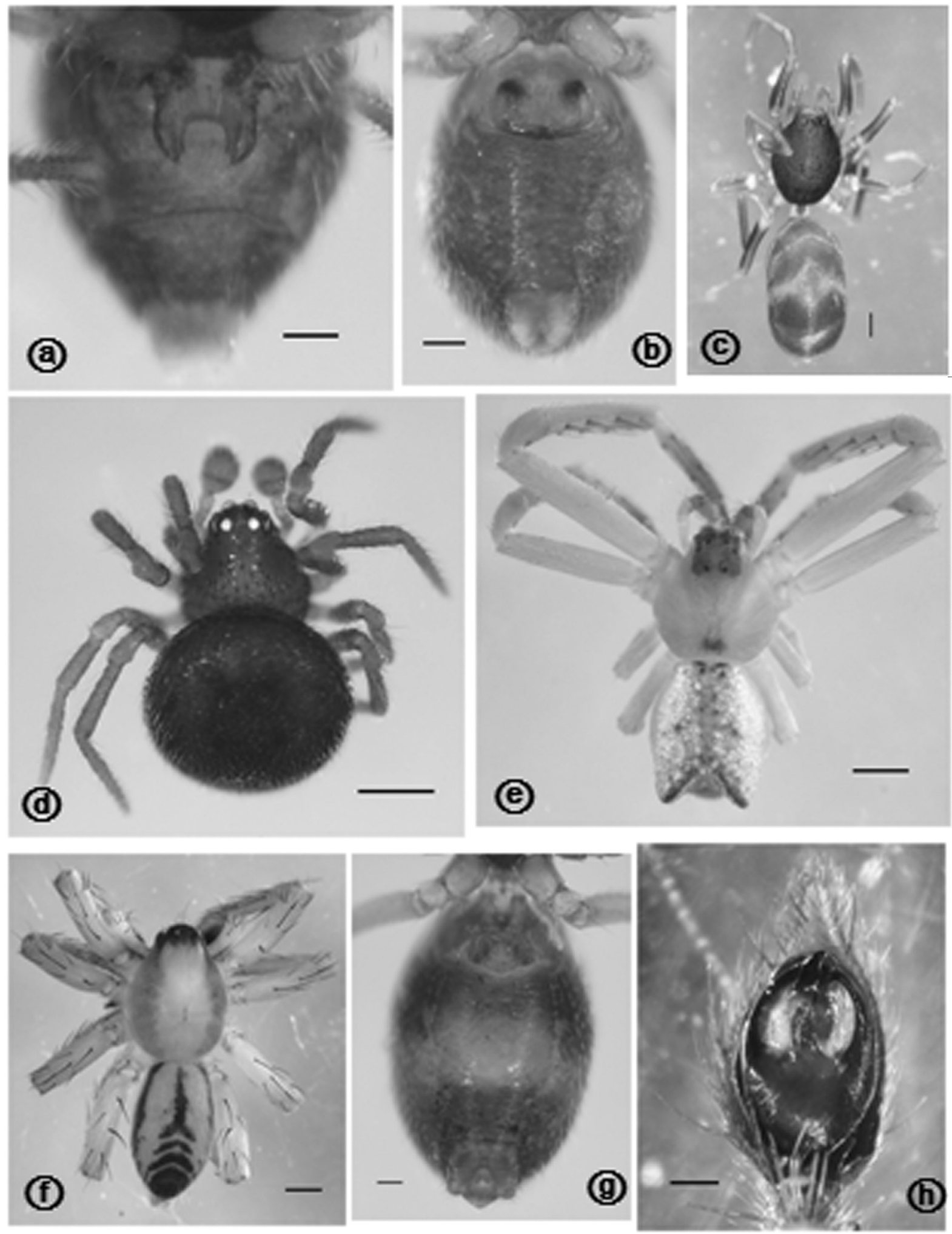

Figura 4. a, Fissiscapus sp. $\bigcirc$ epigineo; b, Paraletes sp. $\bigcirc$ epigineo; c, Pescennina sp. $\bigcirc$ habitus; d, Wirada sp. $\widehat{O}$ habitus; e, Sidymella sp. ninfa habitus; f, Josa nigrifrons $\widehat{\partial}$ habitus; g, Tennesseellum formica $\bigcirc$ opistosoma; h, Rualena simplex $\hat{\partial}$ palpo ventral. Escalas: a, $\mathrm{b}, \mathrm{g}=0.1 \mathrm{~mm} ; \mathrm{c}, \mathrm{h}=0.2 \mathrm{~mm} ; \mathrm{d}=0.3 \mathrm{~mm} ; \mathrm{e}, \mathrm{f}=0.5 \mathrm{~mm}$. 

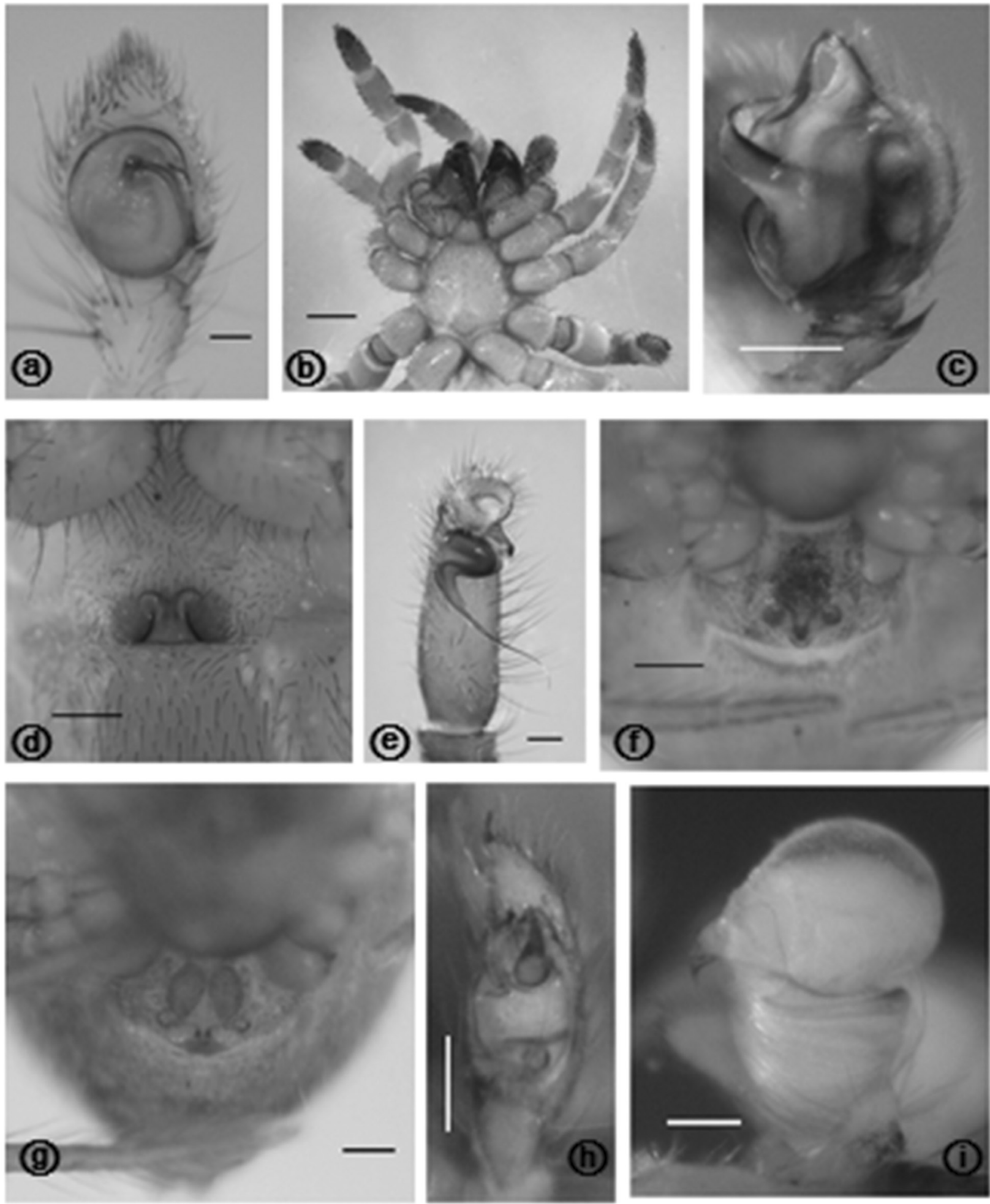

Figura 5. a, Tmarus vitusus $\hat{\jmath}$ palpo ventral; b, Ummidia zilchi $\bigcirc$ prosoma ventral; c, Grammonota teresta $\hat{\jmath}$ palpo retrolateral; d, Leucauge acuminata $\bigcirc$ epigineo; e, Ummidia zebrina $\widehat{o}$ palpo ventral; f, Phycosoma altum $\bigcirc$ epigineo; $\mathrm{g}$, Thymoites puer $q$ epigineo;

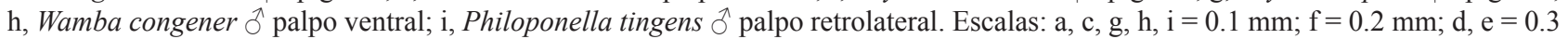
$\mathrm{mm} ; \mathrm{b}=1 \mathrm{~mm}$. 

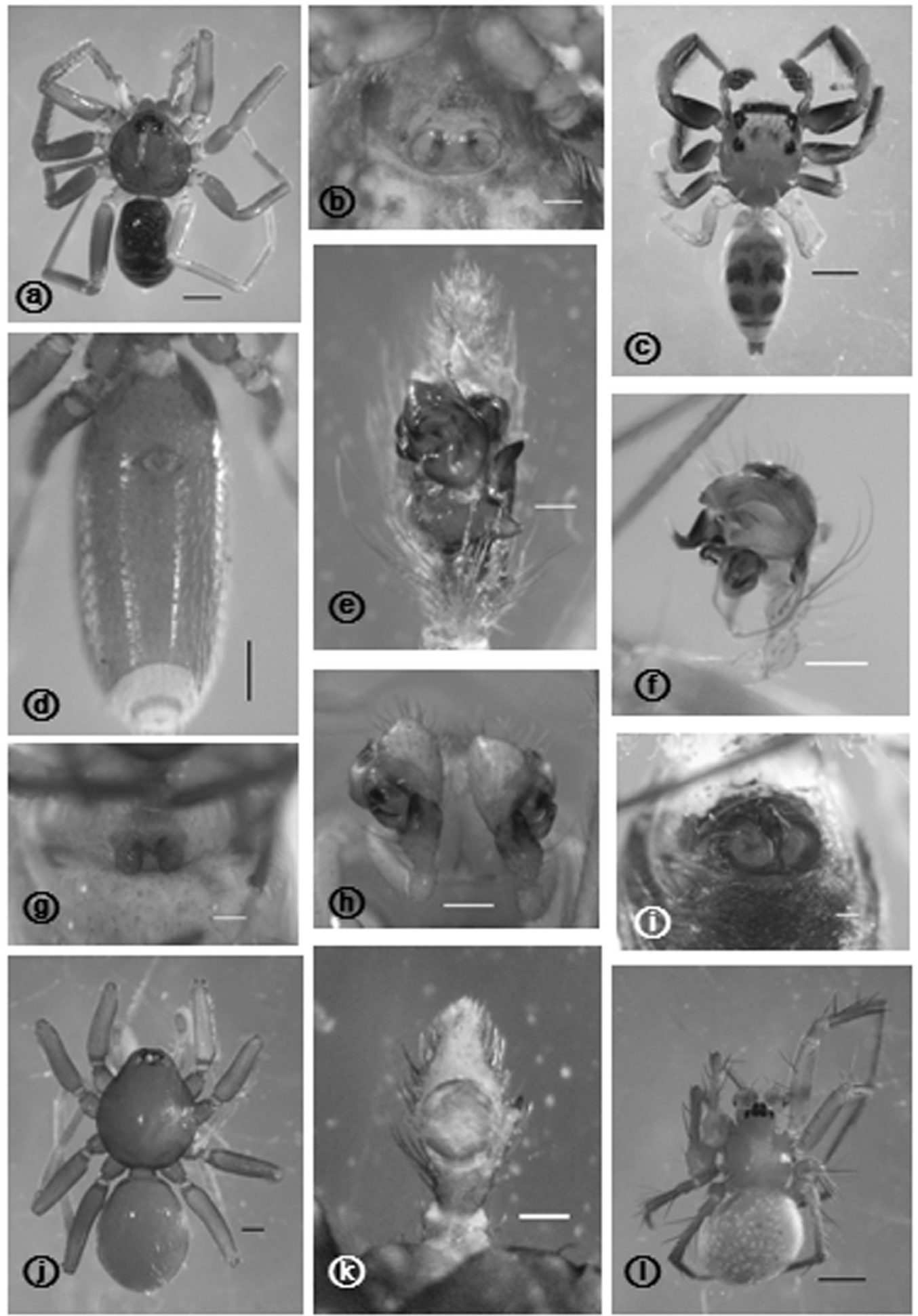

Figura 6. a, Piabuna sp. $\widehat{\delta}$ habitus; b, Naphrys sp. + epigineo; c, Paramarpissa sp. $\widehat{\delta}$ habitus; d, Scaphiella cocona $q$ opistosoma ventral; e, Melpomene transversa ô palpo ventral; f, Selenyphantes longispinosus đ̊ palpo retrolateral; g, Leucauge simplex + epigineo;

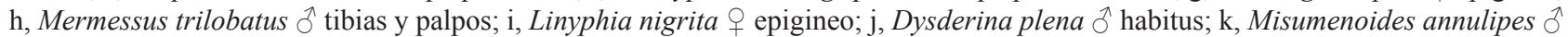
palpo ventral; 1, Mangora ixtapan $\widehat{\partial}$ habitus. Escalas: $\mathrm{i}, \mathrm{k}=0.1 \mathrm{~mm} ; \mathrm{b}, \mathrm{d}, \mathrm{e}, \mathrm{f}, \mathrm{g}, \mathrm{h}, \mathrm{j}=0.2 \mathrm{~mm} ; \mathrm{a}, \mathrm{l}=0.5 \mathrm{~mm} ; \mathrm{c}=1 \mathrm{~mm}$. 
evidenciando que se realizó un esfuerzo de muestreo comparable. En el nivel de familia, la jerarquía de riqueza de especies es muy similar a la registrada en otros estudios de zonas tropicales, donde Theridiidae resultó la más diversa (Sørensen et al., 2002; Sørensen, 2004) o en los 2 primeros lugares de riqueza junto con Araneidae (Silva y Coddington, 1996). Linyphiidae es poco diversa en regiones tropicales bajas, pero algunos estudios sugieren que incrementa su importancia con la altitud (Russell y Stork, 1994; Sørensen, 2004), como ocurre en el presente estudio. A pesar de que en el sotobosque se encontró menor número de familias que en el suelo, el primer estrato posee mayor número de especies, debido principalmente a una mayor diversidad en Theridiidae, Araneidae, Tetragnathidae y Uloboridae, las cuales son arañas tejedoras de red que se caracterizan por su abundancia en la vegetación. Por otro lado, la familia Salticidae (no tejedora de redes) fue diversa en ambos estratos, con una riqueza similar de especies. Entre los géneros, cabe destacar la riqueza de Anyphaena Sundevall, 1833 (Anyphaenidae), del cual se registraron 11 especies en los 2 estratos, contrastando con la mayoría de los otros géneros que sólo tuvieron 1 o 2 especies ( $91 \%$ del total de géneros).

Se han registrado previamente diferencias en riqueza de especies entre estratos de BMM (Sørensen, 2003; Yanoviak et al., 2003). Entre los estratos, Sørensen (2003) obtuvo una complementariedad de $58.1 \%$, mientras que nuestros resultados muestran un $76.2 \%$. Lo anterior puede indicar diferencias de mayor importancia en las condiciones del microclima de los estratos estudiados en el BMM del volcán Tacaná y por lo tanto el nivel de especificidad de estrato es más alto.

\section{Agradecimientos}

A José Álvaro García Ballinas, José Antonio López Méndez, Estela Sentíes Celma y Eduardo Rafael Chamé Vázquez (ECOSUR), por colaborar en la recolecta de los ejemplares. A José Álvaro García Ballinas, Erika Orozco Díaz, Sergio Dorian Moreno Mendoza y Kaleb Zárate Gálvez (Universidad de Ciencias y Artes de Chiapas), por su ayuda en la separación de las muestras. Al Consejo Nacional de Ciencia y Tecnología (CONACYT), por el apoyo al proyecto 52923 (2007) y por las becas otorgadas a Julieta Maya-Morales y a David Chamé-Vázquez. Se agradece también a los evaluadores anónimos por sus valiosos comentarios y sugerencias.

\section{Literatura citada}

Acosta, S. 2004. Afinidades de la flora genérica de algunos bosques mesófilos de montaña de nordeste, centro y sur de
México: enfoque fenético. Anales del Instituto de Biología, UNAM, Serie Botánica 75:61-72.

Álvarez, F. 1999. Estudio faunístico de la familia Araneidae (Arachnida: Araneae); en la selva baja caducifolia del municipio de "El Limón", Jalisco, México. Tesis, Escuela Nacional de Estudios Profesionales, Campus Iztacala, Universidad Nacional Autónoma de México, Tlalnepantla, Estado de México. 87 p.

Anderson, R. S. y J. S. Ashe. 2000. Leaf litter inhabiting beetles as surrogates for establishing priorities for conservation of selected tropical montane cloud forests in Honduras, Central America (Coleoptera: Staphylinidae, Curculionidae). Biodiversity and Conservation 9:617-653.

Bubb, P., I. May, L. Miles y J. Sayer. 2004. Cloud Forest Agenda. UNEP-WCMC, Cambridge. 32 p.

Cardoso, M. D. 1979. El clima de Chiapas y Tabasco. Universidad Nacional Autónoma de México, México D. F. 99 p.

Castelo, J. L. 2000. Diversidad de Salticidae (Arachnida: Araneae) en una localidad de selva baja caducifolia del sur de Jalisco, México. Tesis, Escuela Nacional de Estudios Profesionales, Campus Iztacala, Universidad Nacional Autónoma de México. Tlalnepantla, Estado de México. 181 p.

Collwell, R. K. y J. A. Coddington. 1994. Estimating terrestrial biodiversity through extrapolation. Philosophical Transactions of the Royal Society of London series B-Biological Sciences 345:101-118.

Coddington, J. A., C. Griswold, D. Dávila, E. Peñaranda y S. Larcher. 1991. Designing and testing sampling protocols to estimate biodiversity in tropical systems. In The unity of evolutionary biology, vol. 1. Proceedings of the Fourth International Congress of Systematics and Evolutionary Biology, E. Dulley (ed.). Dioscorides, Portland, Oregon, p. 44-60.

Corcuera, P. y M. L. Jiménez. 2009. Diversidad de arañas asociadas al follaje en una selva baja caducifolia de Jalisco. Folia Entomológica Mexicana 47:49-60.

Correa, M. M. 2001. Estudio comparativo de las arañas de la vegetación arbustiva y arbórea de dos comunidades vegetales en Tlancualpican, Puebla y Cerro El Horno, Morelos, México. Tesis, Facultad de Estudios Superiores, Zaragoza, Universidad Nacional Autónoma de México. México, D. F. 45 p.

Durán, C. G. 2000. Estudio faunístico de la familia Theridiidae (Arachnida: Araneae) en la selva baja caducifolia del sur de Jalisco (Mpio. El Limón), México. Tesis, Escuela Nacional de Estudios Profesionales, Campus Iztacala, Universidad Nacional Autónoma de México. Tlalnepantla, Estado de México. 112 p.

García-Villafuerte, M. A. 2009. La araneofauna (Araneae) reciente y fósil de Chiapas, México. Revista Mexicana de Biodiversidad 80:633-646.

Gertsch, W. J. y L. I. Davis. 1940. Report on a collection of spiders from Mexico. III. American Museum Novitates 1069:1-22. 
Ibarra-Núñez, G. 1979. Las arañas Labidognatha de la parte norte del Pedregal de San Ángel. Tesis, Facultad de Ciencias, Universidad Nacional Autónoma de México. México, D.F. $134 \mathrm{p}$.

Ibarra-Núñez, G. y D. Chamé-Vázquez. 2009. Las arañas del suelo del bosque mesófilo de montaña de la Reserva de la Biosfera Volcán Tacaná, Chiapas: efectos de las perturbaciones antropogénicas. In Entomología mexicana, vol. 8, E. G. Estrada, A. Equihua, M. P. Chaires, J. A. Acuña, J. R. Padilla y A. Mendoza (eds.). Sociedad Mexicana de Entomología. p. 47-52.

Jiménez, M. L. 1996. Araneae. In Biodiversidad, taxonomía y biogeografía de artrópodos de México: hacia una síntesis de su conocimiento, J. Llorente, A. N. García y E. González (eds.). Universidad Nacional Autónoma de México, México, D. F. p. 83-101.

Llinas, J. y M. L. Jiménez. 2004. Arañas de humedales del sur de Baja California, México. Anales del Instituto de Biología, Universidad Nacional Autónoma de México, Serie Zoología 75:283-302.

Logunov, D. V. y B. Cutler. 1999. Revision of the genus Paramarpissa F. O. P. Cambridge, 1901 (Araneae, Salticidae). Journal of Natural History 33:1217-1236.

Mallis, R. E. y L. E. Hurd. 2005. Diversity among grounddwelling spider assemblages: habitat generalists and specialists. Journal of Arachnology 33:101-109.

Maya-Morales, J. 2010. Diversidad de arañas de la vegetación en un bosque mesófilo de montaña en Chiapas, México. Tesis, El Colegio de la Frontera Sur, Tapachula, Chiapas. 69 p.

Medina, F. J. 2002. Las arañas y su distribución temporal en un bosque de San Martín Cachihuapan, Municipio de Villa del Carbón, Estado de México. Tesis, Facultad de Estudios Superiores, Campus Iztacala, Universidad Nacional Autónoma de México. Tlalnepantla, Estado de México. 129 p.

Medina, F. J. 2005. Las arañas errantes del manglar de Chiapas, México. Tesis, Facultad de Ciencias, Universidad Nacional Autónoma de México. México, D. F. 120 p.

Myers, N., R. A. Mittermeier, C. G. Mittermeier, G. A. B. de Fonseca y J. Kent. 2000. Biodiversity hotspots for conservation priorities. Nature 403:853-858.

Nieto, I. G. 2000. Inventario de arañas de algunas localidades de los estados de Puebla y Morelos en la parte alta del Balsas. Tesis, Facultad de Estudios Superiores, Zaragoza, Universidad Nacional Autónoma de México. México, D. F. 83 p.

Platnick, N. I. 2010. The world spider catalog, ver. 11.0. American Museum of Natural History. http://research.amnh. org/entomology/spiders/catalog/index.html; última consulta: 8.XII.2010.

Richman, D. B. 1981. A revision of the genus Habrocestum (Araneae, Salticidae) in North America. Bulletin of the American Museum of Natural History 170:197-206.
Rubio, G. D., J. A. Corronca y M. P. Damborsky. 2008. Do spider diversity and assemblages change in different contiguous habitats? A case study in the protected habitats of the Humid Chaco Ecoregion, Northeast Argentina. Environmental Entomology 37:419-430.

Russell, A. y N. E. Stork. 1994. Abundance and diversity of spiders from the canopy of tropical rainforests with particular reference to Sulawesi, Indonesia. Journal of Tropical Ecology $10: 545-558$

Santos, A. 2001. Diversidad de arañas tejedoras asociadas a márgenes de arroyos, en dos localidades de la región del Soconusco, Chiapas; México. Tesis, Facultad de Ciencias, Universidad Nacional Autónoma de México, México, D. F. 123 p.

Santos, A. 2005. Arañas tejedoras asociadas a los manglares de la Costa de Chiapas. Tesis, Facultad de Ciencias, Universidad Nacional Autónoma de México, México, D. F. 137 p.

Schmidt, M. H., Y. Clough, W. Shulz, A. Westphalen y T. Tscharntke. 2006. Capture efficiency and preservation attributes of different fluids in pitfall traps. Journal of Arachnology 34: 159-162.

SPP (Secretaría de Programación y Presupuesto).1981. Carta de climas. Villahermosa. Escala 1: 1,000,000 Coordinación General de los Servicios Nacionales de Estadística, Geografía e Informática, México, D. F.

Silva, D. y J. A. Coddington. 1996. Spiders of Pakitza (Madre de Dios, Peru): species richness and notes on community structure. In Manu - The biodiversity of southeastern Peru, D. E. Wilson y A. Sandoval (eds.). Smithsonian Institution, Washington, D.C. p. 253-311.

Sørensen, L. L. 2003. Stratification of the spider fauna in a Tanzanian forest. In Arthropods of tropical forest: spatiotemporal dynamics and resource use in the canopy, Y. Basset, V. Novotny, S. E. Miller y R. L. Kitching (eds.). Cambridge University Press. p. 92-101.

Sørensen, L. L. 2004. Composition and diversity of the spider fauna in the canopy of a montane forest in Tanzania. Biodiversity and Conservation 13:437-452.

Sørensen, L. L., J. A. Coddington y N. Scharff. 2002. Inventorying and estimating subcanopy spider diversity using semiquantitative sampling methods in an Afromontane forest. Environmental Entomology 31:319-330.

Toledo, T. 2010. El bosque mesófilo de montaña. In El bosque mesófilo de montaña en México: amenazas y oportunidades para su conservación y manejo sostenible, Comisión Nacional para el Conocimiento y Uso de la Biodiversidad (CONABIO). México D. F. p. 15-17.

Valdez, A., C. G. Durán y J. L. Castelo. 2005. Relaciones zoogeográficas de los géneros de arañas (Arachnida: Araneae) colectados en la selva lacandona; Frontera Corozal, Ocosingo, Chiapas. In Entomología mexicana, vol. 4, A. Morales, A. Mendoza, M. P. Ibarra y S. Standford (eds.). 
Colegio de Posgraduados, Montecillo, Estado de México. p 55-60.

Yanoviak, S. P., G. Kragh y N. M. Nadkarni. 2003. Spider assemblages in Costa Rican cloud forests: effects of forest level and forest age. Studies on Neotropical Fauna and Environment 38:145-154.

Apéndice 1. Lista de especies de arañas del bosque mesófilo de la Reserva de la Biosfera Volcán Tacaná, Chiapas, México. Estrato de recolecta: $\ddagger$, suelo, $\S$, sotobosque. Nuevos registros: ${ }^{1}$ género para México, ${ }^{2}$ especie para México, ${ }^{3}$ género para Chiapas, ${ }^{4}$ especie para Chiapas

\section{AGELENIDAE}

${ }^{3,4}$ Melpomene transversa (F. O. P.-Cambridge, 1902) †

${ }^{2}$ Rualena simplex (F. O. P.-Cambridge, 1902) $\$ \S$

AMAUROBIIDAE

Amaurobiidae sp1 affin gen. Retiro +

ANYPHAENIDAE

Anyphaena trifida F. O. P.-Cambridge, $1900 \ddagger \S$

Anyphaena $\mathrm{sp} 1+\S$

Anyphaena $\mathrm{sp} 2 \ddagger \S$

Anyphaena sp3 $₫ \S$

Anyphaena sp4 §

Anyphaena sp5 §

Anyphaena sp6 §

Anyphaena sp7 $\S$

Anyphaena $\mathrm{sp} 8 \S$

Anyphaena sp9 §

Anyphaena sp10 +

${ }^{1,2}$ Josa nigrifrons (Simon, 1897) §

Wulfila sp1

Wulfila $\mathrm{sp} 2+\S$

ARANEIDAE

Aculepeira $\mathrm{sp} 1$ affin $A$. visite $\S$

Araneus sp1 affin $A$. expletus $\S$

Araneus sp2 affin A. lanio $\S$

Cyclosa sp1 affin C. turbinata $\S$

Cyclosa sp2 §

Cyclosa sp3 affin C. nodosa $\S$

${ }^{4}$ Mangora ixtapan Levi, $2005+\S$

Mangora sp1 affin M. purulha $\$ \S$

Micrathena lenca Levi, $1985+\S$

Micrathena margerita Levi, 1985 §

Ocrepeira rufa (O. P.-Cambridge, 1889) §

Ocrepeira subrufa (F. O. P.-Cambridge, 1904) §

Ocrepeira $\mathrm{sp} 1 \S$

Ocrepeira sp2 affin O. globosa §

CLUBIONIDAE

Elaver $\mathrm{sp} 1+\S$

CORINNIDAE

Creugas sp 1 +

Megalostrata raptor (L. Koch, 1866) $\$ \S$

${ }^{3}$ Piabuna $\mathrm{sp} 1 \ddagger$

Trachelas $\mathrm{sp} 1+\S$

Trachelas $\mathrm{sp} 2 \S$

\section{CTENIDAE}

Ctenus sp $1+\S$

CTENIZIDAE

${ }^{2}$ Ummidia zebrina (F. O. P.-Cambridge, 1897) \$

${ }^{2}$ Ummidia zilchi Kraus, 1955 †

CYBAEIDAE

Cybaeidae sp $1+$

DIPLURIDAE

Euagrus carlos Coyle, 1988 †

GNAPHOSIDAE

Zimiromus sp1 t

LEPTONETIDAE

Darkoneta sp1 +

Darkoneta $\mathrm{sp} 2 \ddagger \S$

LINYPHIIDAE

Ceratinopsis sp $1+$

${ }^{1}$ Fissiscapus $\mathrm{sp} 1$ +

${ }^{2}$ Grammonota teresta Chickering, 1970 §

Jalapyphantes cuernavaca Gertsch y Davis, $1946 \S$

Linyphia linguatula (F. O. P.-Cambridge, 1902) $\$ \S$

${ }^{4}$ Linyphia nigrita (F. O. P.-Cambridge, 1902) §

Linyphia sp1

Linyphia sp2 affin L. duplicata $\S$

Meioneta $\mathrm{sp} 1 \ddagger \S$

Meioneta $\mathrm{sp} 2 \S$

${ }^{4}$ Mermessus trilobatus (Emerton, 1882) $\$ \S$

Mermessus sp1 +

Mermessus $\mathrm{sp} 2+$

${ }^{1}$ Paraletes $\mathrm{sp} 1 \S$

${ }^{1}$ Paraletes $\mathrm{sp} 2+$

${ }^{3,4}$ Selenyphantes longispinosus (O. P.-Cambridge, 1896) §

1,2 Tennesseellum formica (Emerton, 1882) §

Walckenaeria crocea Millidge, $1983+\S$

Erigoninae sp $1+\S$

Erigoninae sp2 affin gen. Asemostera $\ddagger \S$

LYCOSIDAE

Trochosa sp1 t

MIMETIDAE

Mimetus sp $1 \S$

MYSMENIDAE

Calodipoena colima (Gertsch, 1960) $\ddagger \S$

Calodipoena sp1 affin C. stathamae $\S$ 
Apéndice 1. Continúa

Calodipoena $\mathrm{sp} 2 \S$

NESTICIDAE

Gaucelmus augustinus Keyserling, 1884 §

\section{OCHYROCERATIDAE}

Ochyrocera $\mathrm{sp} 1$ †

Speocera sp1 +

OECOBIIDAE

Oecobius concinnus Simon, 1893 †

OONOPIDAE

${ }^{4}$ Dysderina plena O. P.-Cambridge, $1894 \$ \S$

Oonops sp1 †

${ }^{1}$ Pescennina $\mathrm{sp} 1 \S$

${ }^{3,4}$ Scaphiella cocona Platnick y Dupérré, 2010 \$

Triaeris $\mathrm{sp} 1$ \$

Oonopidae sp1 +

Oonopidae sp 2 \$

PHOLCIDAE

Anopsicus sp1 +

Metagonia asintal Gertsch, $1971 \$ \S$

Modisimus sp1

Pholcidae sp1 §

Pholcidae sp 2 +

SALTICIDAE

Corythalia $\mathrm{sp} 1+$

Euophrys sp1 +

Lyssomanes $\mathrm{sp} 1+\S$

Mexigonus sp1 affin $M$. morosus $\S$

${ }^{3}$ Naphrys sp $1+\S$

${ }^{3}$ Naphrys sp $2 \ddagger \S$

${ }^{3}$ Paramarpissa $\mathrm{sp} 1 \S$

Zygoballus tibialis F. O. P.-Cambridge, $1901 \$ \S$

Euophryinae sp1 $\S$

Euophryinae sp2 §

Euophryinae sp3

Salticidae sp1 $\S$

Salticidae sp2 +

SCYTODIDAE

Scytodes sp $1+\S$

SELENOPIDAE

Selenops sp1 $\S$

SYMPHYTOGNATHIDAE

Symphytognatha $\mathrm{sp} 1 \S$

TETRAGNATHIDAE

Chrysometa brevipes (O. P.- Cambridge, 1889) §

Chrysometa sp1 affin C. universitaria $\$ \S$

Chrysometa sp2 affin C. buga $\S$

Cyrtognatha sp1 $\S$

Glenognatha spherella Chamberlin e Ivie, $1936 \S$

${ }^{2}$ Leucauge acuminata (O. P.-Cambridge, 1889) §
${ }^{4}$ Leucauge simplex F. O. P.-Cambridge, $1903+\S$

Tetragnatha sp1 affin $T$. cognata $\S$

THERAPHOSIDAE

Theraphosidae sp $1 \ddagger$

THERIDIIDAE

Ameridion sp1 affin A. ruinum §

Anelosimus jucundus O. P.-Cambridge, $1896 \S$

Chrosiothes goodnightorum (Levi, 1954) $\$$ §

Cryptachaea $\mathrm{sp} 1 \S$

Dipoena sp1 affin D. insulana $\S$

Exalbidion dotanum (Banks, 1914) §

Faiditus sp1

Hentziectypus florens (O. P.-Cambridge, 1896) $\$ \S$

Phoroncidia triunfo Levi, 1964 §

${ }^{2}$ Phycosoma altum (Keyserling, 1886) §

Spintharus flavidus Hentz, 1850 §

Stemmops questus Levi, 1955 *

Stemmops sp $1+$

Theridion adjacens (O. P.-Cambridge, 1896) §

Theridion evexum Keyserling, 1884 \$

Theridion nudum Levi, 1959 §

Theridion trepidum O. P.-Cambridge, 1898 §

Theridion sp1 $\S$

Theridion $\mathrm{sp} 2$ t

Theridion $\mathrm{sp} 3$ !

${ }^{2}$ Thymoites puer (Mello-Leitão, 1941) $\$ \S$

Thymoites sp1 affin T. expulsus $\$ \S$

Thymoites sp2 affin T. sclerotis $\$$

Thymoites sp3 affin T. orilla *

Tidarren haemorrhoidale (Bertkau, 1880) §

${ }^{2}$ Wamba congener O. P.-Cambridge, $1896 \S$

Wamba sp1 $\S$

${ }^{1}$ Wirada $\mathrm{sp} 1 \S$

Theridiidae sp1 $\S$

THERIDIOSOMATIDAE

Epeirotypus $\mathrm{sp} 1+\S$

Theridiosoma davisi Archer, $1953 \S$

Wendilgarda mexicana Keyserling, $1886 \S$

THOMISIDAE

${ }^{4}$ Misumenoides annulipes (O. P.-Cambridge, 1891) §

${ }^{1}$ Sidymella $\mathrm{sp} 1$ affin $S$. parallela $\S$

${ }^{2}$ Tmarus vitusus Chickering, $1965 \S$

ULOBORIDAE

Miagrammopes sp1 $\S$

Philoponella signatella (Chamberlin e Ivie, 1936) §

${ }^{2}$ Philoponella tingens (Chamberlin e Ivie, 1936) §

Uloborus campestratus Simon, $1893 \S$

ZODARIIDAE

Zodariidae sp1 